\title{
The rates of blindness and of partial sight registration in glaucoma patients
}

\begin{abstract}
Purpose To determine the extent of unregistered blind and partial sight visual loss amongst primary open angle glaucoma (POAG) patients in an outpatient clinic. Methods A 13 year follow-up study was carried out of all patients with POAG attending the outpatient clinics at the Leicester Royal Infirmary during the first 4 months of 1982. Results Ninety (35\%) of 258 patients achieved eligibility for registration and 47 patients $\mathbf{( 1 8 \% )}$ were registered, consisting of 39 who were eligible and 8 who were not. Fifty-seven per cent of eligible patients remained unregistered. Patients with visual loss due to visual acuity loss were much more likely to be registered than patients with either visual field loss $(p<0.001)$ or mixed visual acuity/ visual field loss $(p<0.001)$. All categories of eligible patients experienced a delay between eligibility and registration. This delay was much longer for the visual field loss patients (median delay 61.8 months). Patients with untreatable disease were more likely to be registered. The rates of registration are improving.

Conclusion A large proportion of glaucoma patients who are eligible for registration as either blind or partially sighted remain unregistered. Those who are registered often experience prolonged delays before becoming registered.
\end{abstract}

Key words Blindness, Glaucoma, Registration

The problem of non-registration of eligible patients in the community has long been recognised. ${ }^{1-6}$ Gibson et al. ${ }^{6}$ examined blindness prevalence in an elderly rural population in Leicestershire, and concluded that true prevalence of blindness was underestimated by a factor of 1.1 for blind registration and a factor of 1.5 for partial sight registration. The most recent study of visual disability from the Royal National Institute for the Blind (RNIB) highlighted the extent of unregistered blindness and partial sight within the community. It estimated that only about $31 \%$ of adult patients
A.J.W. KING, A. REDDY, J.R. THOMPSON, A.R. ROSENTHAL eligible for blind registration and $12 \%$ of those eligible for partially sighted registration were registered. ${ }^{1}$ Of those eligible but not registered, $77 \%$ had been seen by an eye specialist at some time in the past, suggesting that some registerable disease had been missed by the examining eye specialist. ${ }^{1}$

The suspicion that eligible patients attending outpatient clinics were not registered was confirmed in two separate studies. ${ }^{7,8}$ These studies identified several risk factors for underregistration of particular interest to the glaucoma population: firstly that patients with permanent visual loss receiving treatment were less likely to be registered than patients with a condition for which there was no treatment; 7,8 and secondly that patients who had visual field loss alone were less likely to be registered than people whose central visual acuity was affected. ${ }^{8}$

To establish to what extent these factors and others contributed to under-registration of blind and partially sighted patients with glaucoma, a cohort of primary open angle glaucoma (POAG) patients attending the Leicester Royal Infirmary were investigated.

\section{Methods}

All patients with POAG who attended the outpatient department of the Leicester Royal Infirmary during the first 4 months of 1982 were included in the study; both newly diagnosed patients and patients attending for review were included in the cohort. The case sheets of these patients were obtained and information about visual acuity and visual field status was recorded for each outpatient visit.

Information on all patients included was available from their first clinic visit (usually at diagnosis) to the end of 1994 or, if the patient was discharged or died before then, until their final visit. The details of patients were checked against the register for blindness at the local branch of the RNIB.

Patients were defined as eligible for registration as blind or partially sighted if their visual acuity was $<6 / 60$, according to guidelines contained on the BD8 registration
A.J.W. King Department of Ophthalmology Queens Medical Centre Nottingham, UK

\section{A. Reddy \\ West Norwich Hospital Norwich, UK}

\section{J.R. Thompson}

A.R. Rosenthal Department of Ophthalmology Clinical Sciences Building Leicester Royal Infirmary Leicester, UK

Anthony King, FRCOphth Department of Ophthalmology Queens Medical Centre Nottingham NG7 2UH, UK

Tel: +44 (0)115 9249924, ext 42530

e-mail:

anthonyjking@hotmail.com

Received: 21 July 1999 Accepted in revised form: 11 February 2000 
399 Registered as Glaucoma

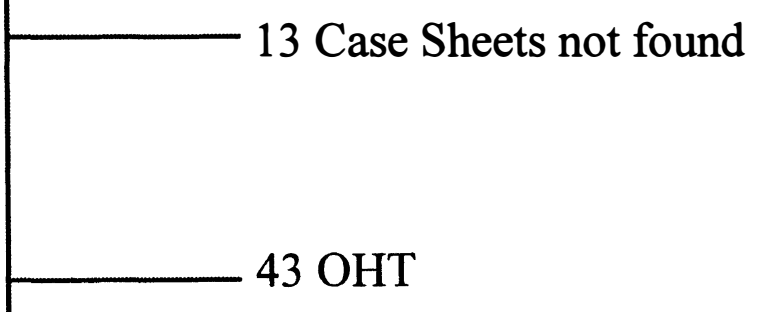

80 Other Glaucoma

5 Non Glaucoma

\section{Confirmed as POAG}

Fig. 1. Diagnoses of patients labelled as 'glaucoma' on the hospital database. OHT, ocular hypertension; POAG, primary open angle glaucoma.

form. Establishing blindness due to visual field loss was more difficult as no strict criteria exist to define the terms moderate and severe visual field contraction which are referred to on the BD8 form. During the time period of this study the primary visual field used for monitoring glaucoma patients was the Goldmann visual field. Visual field assessments for eligibility were made on the basis of a III4e or larger target. Two broad categories of visual field loss were considered severe enough to warrant registration:

1. Severe hemifield loss encroaching to within at least $5^{\circ}$ of fixation in either the horizontal or vertical meridian.

2. Severe visual field constriction to within at least $15^{\circ}$ of fixation in all meridians.

Three categories of registration eligibility were defined:

1. Visual acuity (VA) eligibility: if the best corrected visual acuity was $<6 / 60$ in the better eye.

2. Visual field (VF) eligibility: if there was severe bilateral visual field loss fitting into one of the categories above.

3. Mixed eligibility: if the best corrected visual acuity was $<6 / 60$ in one eye and there was severe visual field loss in accordance with the definitions above in the other eye.

For this study patients were considered as registerable or non-registerable. No distinction was made between partial sight and blind registration, as the main concern of this study was to assess the degree of unregistered eligible visual loss, into which both of these registration categories fall. This study was aimed at identifying only subjects with permanent visual loss who were not registered. To minimise the chance of including patients with reversible visual loss or temporary fluctuating

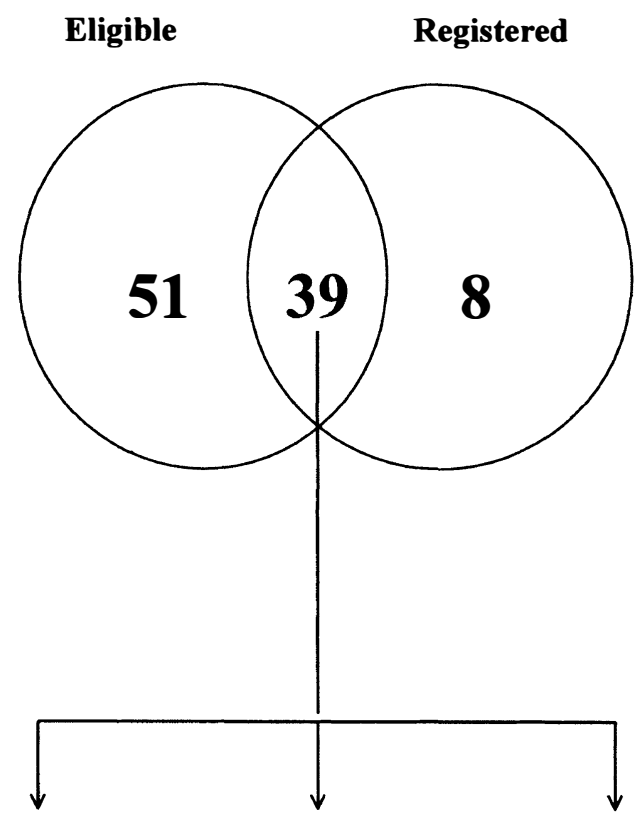

Registered before Eligible

Eligible and Registered at the same time

Eligible before Registered

$\mathbf{N}=\mathbf{8}$

$\mathbf{N}=6$

$\mathbf{N}=\mathbf{2 5}$

Fig. 2. Relationship between eligible and registered patients.

visual loss to a registerable level, subjects were only considered to be eligible for registration if their visual loss lasted for at least 6 months or for three consecutive clinic visits, whichever was longer.

The patient's follow-up period was considered to be up to the date of discharge from the clinic, failure of a patient to attend a clinic or until 31 December 1994, whichever came first. Subjects who were eligible for registration but not registered, or registered but not eligible for registration, at the end of the follow-up period were censored (that is no assumptions can be made about their progression to blindness or eligibility status following the end of follow-up).

For patients who achieved eligibility status prior to their date of blind or partial sight registration the time delay was taken as the time between the date of achieving eligibility status and the date of blind or partial sight registration. Where patients achieving eligibility status were discharged or failed to attend after achieving eligibility criteria but before registration, the time delay to registration was taken as the time they continued to attend clinic up to their final attendance date. If they were not registered as blind before the final follow-up date (31 December 1994) this was considered their final attendance date. Therefore delay in registration represents the time that patients continued to attend the clinic after achieving eligibility status.

Patients who achieved eligibility status were classified as having either treatable or untreatable disease responsible for their visual loss. A disease was considered to be treatable if current treatment, either medical or surgical, was possible (i.e. glaucoma, cataract). If no treatment was available for the disease process (i.e. age-related macular degeneration, ischaemic 
Table 1. Time in months (to the nearest whole month) between eligibility and registration dates $(n=n u m b e r)$

\begin{tabular}{lcccccc}
\hline Group & $n$ & Minimum & First quartile & Median & Third quartile & Maximum \\
\hline Eligible only & 51 & 0 & 12 & 38 & 60 & 173 \\
Eligible before registered & 25 & 1 & 8 & 16 & 40 & 211 \\
Eligible and registered at the same time & 6 & - & - & - & - & - \\
Registered before eligible & 8 & 16 & 19 & 23 & 44 & 97 \\
Registered only & 8 & 12 & 54 & 100 & 132 & 139 \\
\hline
\end{tabular}

optic neuropathy), the condition was considered untreatable. Where the mechanism of visual loss between the two eyes differed the patient's condition was defined as treatable or untreatable on the basis of the condition causing visual loss in the second of the eyes to lose vision to a registerable level.

The times from eligibility to registration were plotted as Kaplan-Meier curves and the significance of predictive factors was assessed by Weibull regression. ${ }^{9}$ Treating the predictive factors as grouped or continuous variables did not materially affect the analysis and the grouped results are presented. For this data set, groups were defined according to the median value of the variable being assessed, thus dividing the data for that variable into groups of equal sizes and increasing the likelihood of detecting statistical significance.

\section{Results}

In all, 399 patients were registered as having glaucoma and attending the outpatient clinic at the Leicester Royal Infirmary during the first 4 months of 1982. Of these patients the case notes of 386 were found. Following review of the case notes, 258 patients were considered to have primary open angle glaucoma (POAG) on the basis of the criteria used. The remaining 128 patients were excluded from the study. Of these remaining patients 43 were ocular hypertensives (OHT), 12 had normal tension glaucoma (NTG), 35 had angle closure glaucoma (ACG), 11 aphakic glaucoma, 8 had secondary glaucoma and 14 other glaucoma (4 pigment dispersion, 3

pseudoexfoliation, 4 narrow angle, 1 congenital and 2 unknown glaucoma). (Fig. 1).
Of the 258 patients with POAG included in the study 128 patients were male and 130 were female. The mean age for the diagnosis of POAG was 69.2 ( \pm 9.9$)$ years, the mean age of eligibility for registration was $76.7( \pm 7.9)$ years, the mean age for registration as blind or partially sighted was $77.4( \pm 8.9)$ years, and the median hospital follow-up time was 130.5 months following diagnosis.

In all $47(18.2 \%)$ patients from this cohort had been registered as blind or partially sighted. Using the criteria defined for eligibility for registration, 90 (34.9\%) patients were eligible for registration. There was an overlap of 39 patients between these two groups (Fig. 2).

Of the patients common to both groups 25 patients were registered after they became eligible (median time 16 months following eligibility; range 1-211 months), 8 patients were registered before they became eligible (median time 23 months before eligibility; range 16-97 months) and 6 were registered at the appropriate eligibility time. Of the 90 patients who became eligible 51 $(56.6 \%)$ were never registered. The median follow-up for these unregistered patients following their eligibility date was 38 months up to the end point of their follow-up (Table 1).

The time to registration following eligibility for the whole group is shown in Fig. 3 and demonstrates the prolonged delay to registration experienced by the majority of eligible patients. The median delay between eligibility and registration for the group as a whole was 37 months.

Of the total of 90 patients who achieved eligibility, 27 $(30 \%)$ were on the basis of visual acuity (VA) criteria, 32 (36\%) visual field (VF) criteria and 31 (34\%) mixed

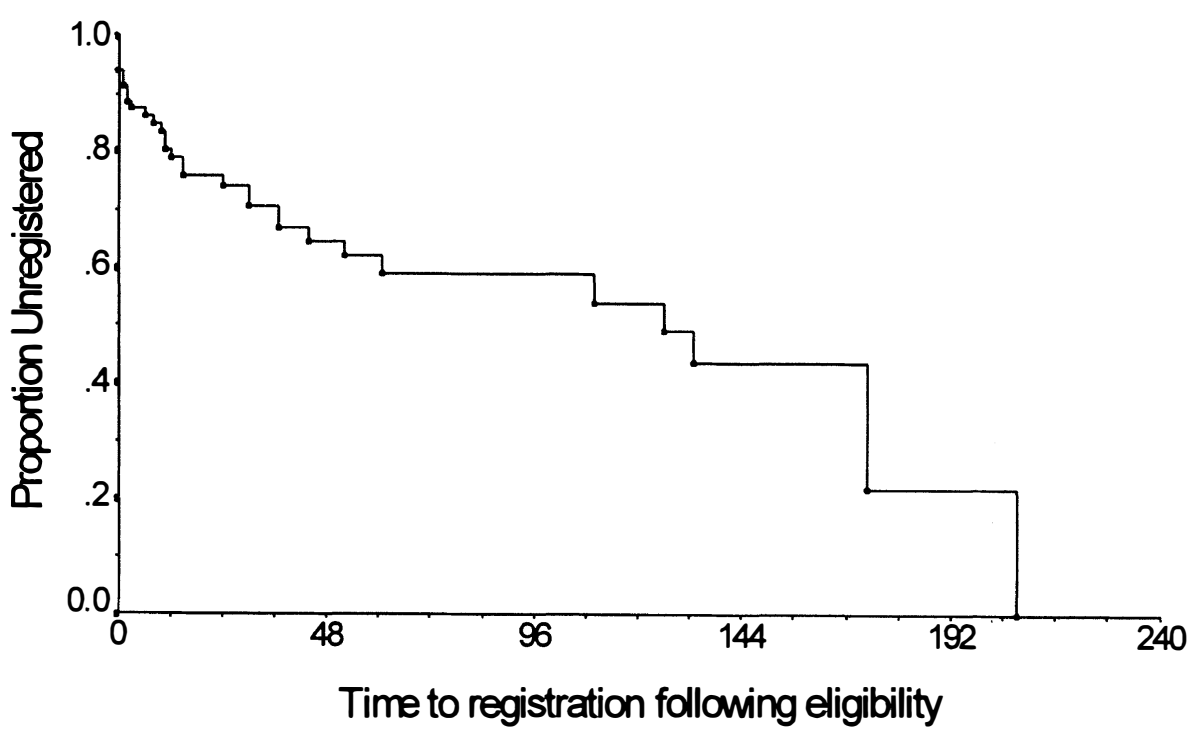

Fig. 3. Kaplan-Meier plot showing time (in months) to registration as blind or partially sighted following eligibility. 
Table 2. Time in months (to the nearest whole month) between eligibility and registration dates for different eligibility standard criteria

\begin{tabular}{|c|c|c|c|c|c|c|}
\hline Group & $n$ & Minimum & First quartile & Median & Third quartile & Maximum \\
\hline \multicolumn{7}{|l|}{ Visual acuity } \\
\hline Eligible only & 8 & 0 & 11 & 25 & 48 & 164 \\
\hline Eligible before registered & 10 & 2 & 7 & 14 & 31 & 53 \\
\hline Eligible and registered at the same time & 5 & - & - & - & - & - \\
\hline \multicolumn{7}{|l|}{ Visual field } \\
\hline Eligible only & 24 & 0 & 9 & 38 & 56 & 173 \\
\hline Eligible before registered & 7 & 1 & 17 & 62 & 129 & 177 \\
\hline Eligible and registered at the same time & 1 & - & - & - & - & - \\
\hline \multicolumn{7}{|l|}{ Mixed } \\
\hline Eligible only & 19 & 6 & 21 & 53 & 80 & 157 \\
\hline Eligible before registered & 8 & 1 & 6 & 13 & 35 & 211 \\
\hline Eligible and registered at the same time & 0 & - & - & - & - & - \\
\hline
\end{tabular}

criteria. Of these, 8 of $27(29.6 \%)$ in the VA group, 24 of 32 $(75 \%)$ in the VF group and 19 of $31(61.3 \%)$ in the mixed group remained unregistered.

For the patients who achieved the eligibility standard prior to or at the same time as registration $(n=82)$, in 23 this was based on VA criteria, in 32 on VF criteria and in 27 on mixed criteria. Of these, $65 \%(15 / 23)$ of patients achieving eligibility by VA criteria were registered as blind or partially sighted, compared with only $25 \%$ $(8 / 32)$ of the VF criteria and $30 \%(8 / 27)$ of the mixed criteria patients (Table 2).

For patients eligible but not registered the VA, VF and mixed criteria groups showed differing median followup times of 25, 38 and 53 months respectively following eligibility before registration (Table 2). For the patients who were eligible and registered in the VA and mixed groups the median time to registration following eligibility was 14 and 13 months respectively, compared with 62 months for the VF group.

Patients in the VA group were much more likely to be registered than those in either the $\operatorname{VF}(p<0.001)$ or mixed criteria groups $(p<0.001)$ (Table 3$)$. These trends are clearly demonstrated on the survival analysis curve, which shows that patients with eligibility due to VA were more likely to be registered and registered early (Fig. 4).

Patients who became eligible after 1983 were more likely to be registered than those becoming eligible before this time $(p<0.001)$. The rates of registration were borderline different between men and women achieving eligibility $(p=0.06)$. There was no influence from age of diagnosis on the rate of registration $(p=0.95)$, or the year of diagnosis comparing those diagnosed before or during 1978 with those diagnosed after $1978(p=0.60)$. Using the Townsend postal code classification for social class it was found that registration rates were unaffected by social class $(p=0.28)$. Considering the disease causing visual loss as treatable or untreatable showed that patients with untreatable disease were more likely to be registered than patients considered to have treatable disease $(p=0.05)$.

Three patients who were offered registration were recorded in the notes to have refused it. All these patients were discharged at the offer visit. One patient who was registered as blind had subsequent sight-improving treatment (cataract extraction) but was not removed from the register.

\section{Discussion}

Visual disability severely restricts an individual's independence. Many of the visually disabled are elderly, or have other disabilities which further restrict their quality of life. ${ }^{1}$ Registration as blind or partially sighted is a voluntary act and in the majority of cases this process is initiated by a consultant ophthalmologist in a hospital environment. Registration of the patient initiates access to a spectrum of support services, which although available to all visually impaired people would often not be directed thus if the patient were not registered.

Table 3. The significance of factors potentially related to registration time following eligibility. Multivariate values are corrected for all other terms

\begin{tabular}{lcccc}
\hline & \multicolumn{2}{c}{ Univariate } & \multicolumn{2}{c}{ Multivariate } \\
\cline { 2 - 5 } Factors compared & Hazard ratio & $p$ value & Hazard ratio & $p$ value \\
\hline Gender (female vs male) & 0.93 & 0.84 & 0.46 & 0.06 \\
Age at diagnosis ( $\leqslant 70$ vs $>$ 70 years) & 0.74 & 0.64 & 0.98 & 0.95 \\
Year of diagnosis ( $\leqslant$ 1978 vs $>$ 1978) & 1.02 & 0.95 & 0.80 & 0.60 \\
Year of eligibility (> 1983 vs $\leqslant 1983)$ & 2.47 & 0.01 & 3.83 & $<.001$ \\
Townsend deprivation score & 1.71 & 0.14 & 0.39 & 0.28 \\
Treatable vs non-treatable & 0.69 & & & 0.05 \\
Reason for eligibility & & & 0.14 & $<0.001$ \\
VF vs VA eligibility & 0.24 & $<0.001$ & 0.26 & $<0.001$ \\
Mixed vs VA eligibility & 0.26 & & & \\
\hline
\end{tabular}

VA, visual acuity; VF, visual field. 


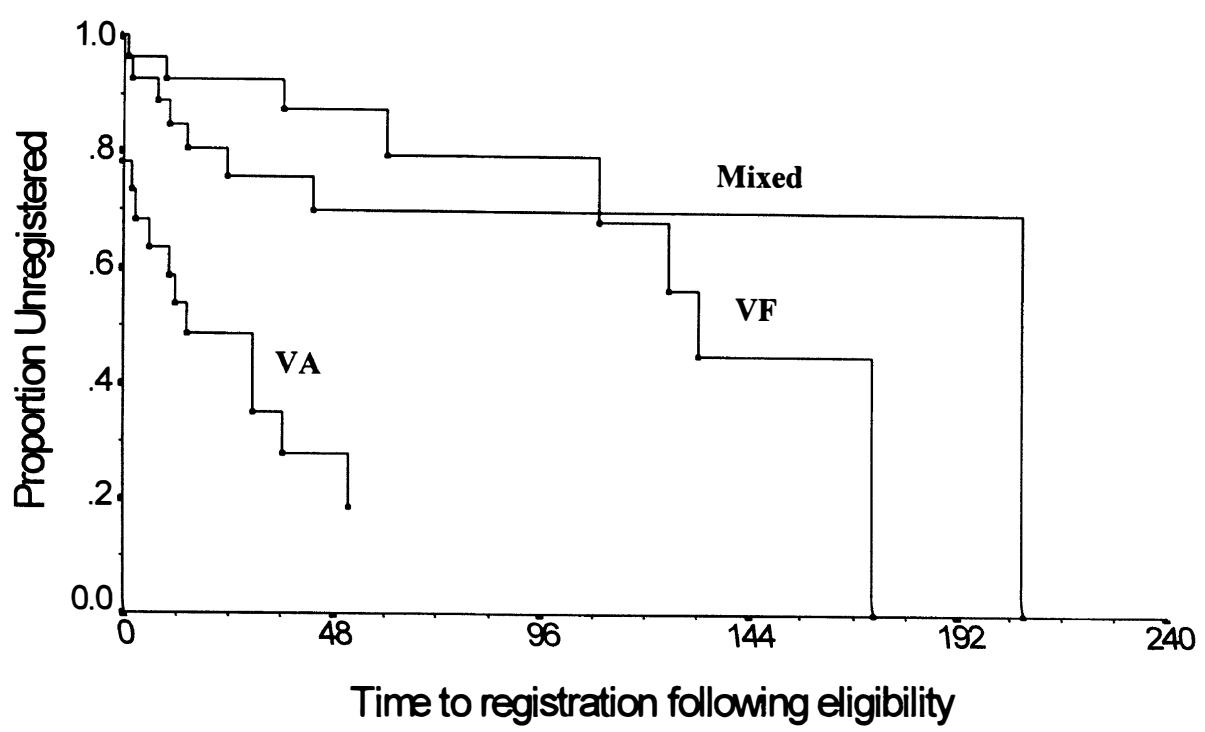

Fig. 4. Kaplan-Meier plot showing time (in months) to registration following eligibility for the different eligibility criteria. VA, visual acuity; VF, visual field.

The aim of this study was to identify the amount of unregistered visual loss within the glaucoma population attending an outpatient department. No distinction between blind and partial sight registration was made as registration into either category triggers access to a range of support services which should benefit the registered patient. Our findings show that over half $(56.6 \%)$ the glaucoma patients who were attending the outpatient clinic who were eligible for blindness or partial sight registration were not registered. This proportion of eligible but unregistered patients is similar to that found in the West Midland ${ }^{7}$ (48.8\%) and London ${ }^{8}$ (51\%) studies, which estimated unregistered blindness in the general outpatient environment. This finding suggests no special effort in identifying eligible patients amongst the glaucoma population, and may be a consequence of many of these glaucoma patients being seen in a general outpatient environment rather than in a specialist glaucoma clinic. We identified a median delay of 37 months for the group as a whole between their eligibility and registration dates. This indicates that not only were a large proportion of eligible patients unregistered, but those who were registered often experienced a considerable delay following their eligibility date before being registered.

Blindness registration may be defined on the basis of either VA or VF loss. In this study a greater proportion of patients were eligible for registration by VF loss criteria alone: $36 \%$ as compared with $10 \%$ and $28 \%$ for the two centres included in the London study. ${ }^{8}$ This can be attributed to the greater degree of pure VF vision loss amongst patients with glaucoma. Bunce et al. ${ }^{8}$ estimated that patients eligible on the basis of VF loss were 3 times less likely to be registered than patients eligible by VA loss, and this is supported by this study. Of the patients registered at or after the time of eligiblity only $35 \%$ of VA patients were not registered compared with $75 \%$ of $\mathrm{VF}$ patients and $70 \%$ of mixed criteria patients. These findings are highly significant, with patients achieving eligibility by VA criteria being more likely to be registered than patients achieving the eligibility standard by either VF $(p<0.001)$ or mixed criteria $(p<0.001)$. In addition, for those patients who are eligible and registered the delay between eligibility and registration is much less for the VA loss patients (median 14.0 months) than for VF loss patients (median 62 months). Therefore, as well as being less likely to be registered, patients who achieved eligibility for registration by VF loss were also much more likely to experience a prolonged delay before registration following their eligibility date. One possible explanation for this is that blindness from VA loss is 'more obvious' than blindness from VF loss; thus the consulting ophthalmologist may have been quicker to identify and register patients blind from VA loss than from VF loss.

The median delay between eligibility and registration for the mixed loss group (median 13 months) was similar to that of the VA group. This suggests that even though only a small proportion of these patients were registered, those who were, were registered early, possibly due to recognition of poor VA in one of their eyes.

Delay in registration may be attributed to fluctuation in the visual function of an individual and in such cases delay in registration is understandable. In this study efforts were made to eliminate this problem and include only patients with stable visual loss for at least 6 months; thus the delay found represents the delay experienced by individuals with continuous visual loss at a registerable level. This delay in registration further adds to the poor quality of care already recognised in failure to register many eligible subjects as blind or partially sighted.

Patients achieving eligibility prior to 1983 were less likely to be registered than patients achieving eligibility after this time. This finding suggests an improvement in identification of patients eligible for registration within the outpatient department. This observation supports previous work by Thompson et al. ${ }^{10}$ who showed that the registration rates in Leicestershire improved between the 
years 1965 and 1985. They suggested that this change was due to a combination of changes in registration practice and a greater awareness of registration facilities among patients. Guidelines issued by the Royal College of Ophthalmologists ${ }^{11}$ should increase awareness and help to accelerate this trend even further.

Previous investigators have suggested that patients with treatable disease are less likely to be registered than patients with untreatable permanent visual loss. ${ }^{7,8}$ This study shows that those patients suffering from glaucoma who developed visual loss due to some other untreatable condition were more likely to be registered than patients who became blind from their glaucoma. The likely explanation for this is that for treatable diseases the focus of the clinician is on treatment and registration only becomes an option when the treatment had failed. For many conditions, including glaucoma, the patient achieves eligibility for registration while treatment is in progress, and these patients, while often continuing to attend regular outpatient follow-up, are not obtaining the benefits that registration may provide.

In this study 16 patients became registered as blind or partially sighted prior to achieving eligibility status. Subsequently 8 of these achieved the eligibility standard but the remaining 8 failed to do so during the period of the study follow-up. Gibson et al. ${ }^{6}$ commented in their community-based study that $27 \%$ of patients registered did not achieve registration criteria. These observations may be due to the varying interpretation of registration levels by the examining ophthalmologists, which may vary considerably between individuals and over time. Evans and Wormald ${ }^{12}$ showed in their study that while there has been little change in the overall rates of registration since 1975 the pattern of registration has changed. There was an increase in partial sight registration and a decrease in blindness registration which, they suggest, results from a change in the interpretation of the cut-off between blindness and partial sight during this period.

The benefits received by blind and partially sighted people differ, blind registered people receiving more extensive benefits with arguably more effect on improvement in quality of life. It is possible that this factor influences ophthalmologists when considering registration of an individual. In cases where it is felt that assistance is definitely warranted, the ophthalmologist may be inclined to register the patient as blind or partially sighted even if they fail to achieve registration levels and thus accelerate the patient's access to support services and facilities. Similarly, patients with significant visual loss such as upper hemifield loss but good preservation of inferior visual field and visual acuity who are relatively asymptomatic may be left unregistered with little or no effects on their quality of life and preservation of local authority resources for patients with more severe visual handicap. Wormald and Evans ${ }^{13}$ have suggested that partial sight registration should be used as a catchall for those in need of social service support. The finding that more than $30 \%$ of the patients in this study were registered before they achieved eligibility levels suggests that to some extent this is already the case.

This study identified 3 patients who had been offered registration but refused it. This is a retrospective study and the reasons for refusal are unknown; similarly we cannot be certain of the exact number of patients who were offered but refused registration as we are relying on a record of this being made in the case notes. If some patients had been offered registration but refused, this may result in an over-estimation of the failure of clinic services to identify and provide access to registration services. Refusal of registration has previously been noted. The postal survey by Graham et al. ${ }^{3}$ found that many of the patients eligible for registration opposed it because they felt it represented a form of charity. In the Birmingham study ${ }^{7} 12 \%$ of patients offered registration refused it.

One patient who had been registered as blind had treatment (cataract surgery 7 years after registration) that improved their vision above the level necessary for registration. This patient was not removed from the register. Aclimandos and Galloway ${ }^{14}$ found in Nottingham that about $5 \%$ of patients registered as blind and $18 \%$ of those registered as partially sighted were registered due to cataract, and that these patients are often placed on the register temporarily prior to surgery and theoretically removed once surgery has been completed.

The definitions of eligibility for registration chosen for this study were aimed at including patients who would be included in the umbrella guidelines provided by the BD8 form. It could be argued that a small number of patients included under these study definitions (hemifield loss in opposite hemifields in different eyes or good central visual acuity with severe visual field loss in one eye and poor central visual acuity with a full visual field in the other eye) may not be eligible for registration. None of the patients in this study fell into either of these categories. This study does not consider patients with mixed visual acuity and visual field loss in the same eye. The degree of mixed loss required for registration is more difficult to define. It is thus possible that we underestimated the number of patients who were eligible for blind or partially sighted registration.

In 1991 a new BD8 form was introduced. Patients are no longer registered but are certified for registration, these certified patients then being placed on the local register by the local authority. The new BD8 form consists of a separate 'part 5' filled in at the time of certification which is sent directly to the Office of Population Census and Surveys (OPCS), this information being entered onto an anonymous database - the Blind and Partially Sighted Survey. This change in procedure should ensure that the OPCS is informed about all certified subjects and that accurate information about blind and partial sight registration rates is available in the future. 
The results of the RNIB study in $1991^{1}$ suggested significantly more unregistered visual disability than had previously been estimated in the community. Of those eligible but not registered, $77 \%$ had been seen by an eye specialist at some time in the past, suggesting that some registerable disease had been missed by the examining eye specialist. The RNIB study was aimed at addressing the needs of visually disabled subjects in the UK. It used a visual acuity cut-off of 6/24 (and >6/24 in some cases), which is much lower than the levels suggested by the BD8 guidelines in the absence of a severe visual field defect. This survey, therefore, while assessing the degree of visual disability within the community, used wider defining criteria for registration than most ophthalmologists would use to certify patients as blind or partially sighted and may therefore overestimate the degree of unregistered visual loss according to the guidelines suggested on the BD8 form. The failure of ophthalmic services to identify patients eligible for registration is without doubt poor, but may not be quite as poor as the RNIB survey would suggest.

\section{Conclusion}

A significant amount of unregistered blindness exists in the outpatient glaucoma population. Patients are more likely to be unregistered if they achieve eligibility by visual field loss. Patients with all eligibility criteria experience a delay in registration from their eligibility date but this is much longer for patients with visual field loss. There is a suggestion that a trend towards less unregistered eligibility is occurring. A combination of improved training to increase awareness of under- registration and increased vigilance in the outpatient clinic may reduce the amount of eligible visual loss that is overlooked.

\section{References}

1. Bruce I, McKennell A, Walker E. Blind and partially sighted adults in Britain: the RNIB survey. Vol 1. London: HMSO, 1991

2. Sorsby A. Blindness and partial sight in England and Wales 1969-1976. Rep Health Soc Subj 129. London: HMSO, 1976.

3. Graham PA, Wallace J, Welsby E, Grace HJ. Evaluation of postal detection of registerable blindness. Br J Prev Soc Med 1968;22:283-41.

4. Brennan ME, Knox EG. An investigation into the purposes, accuracy and effective uses of the blind register in England. Br J Prev Soc Med 1973;27:154-9.

5. Cullinan TR. The epidemiology of visual disability. Health Services Research Unit Report no. 28. London: HMSO, 1977.

6. Gibson JM, Rosenthal AR, Lavery J. A study of the prevalence of eye disease in the elderly in an English community. Trans Ophthalmol Soc UK 1985;104:196-203.

7. Robinson R, Deutsch J, Jones HS, et al. Unrecognised and unregistered visual impairment. Br J Ophthalmol 1994;78:736-40.

8. Bunce C, Evans J, Fraser S, Wormald R. BD8 certification of visually impaired people. Br J Ophthalmol 1998;82:72-6.

9. Cox DR, Oakes D. Analysis of survival data. London: Chapman \& Hall, 1984:chap 3.

10. Thompson JR, Du L, Rosenthal AR. Recent trends in the registration of blindness and partial sight in Leicestershire. Br J Ophthalmol 1989;73:95-9.

11. Registration and rehabilitation of the visually handicapped. London: Royal College of Ophthalmologists, 1994.

12. Evans JR, Wormald RPL. Epidemiological functions of BD8 certification. Eye 1993;7:172-9.

13. Wormald R, Evans J. Registration of blind and partially sighted people. Br J Ophthalmol 1994;78:733-4.

14. Aclimandos WA, Galloway NR. Blindness in the city of Nottingham (1980-1985). Eye 1988;2:431-4. 